\title{
An algorithm for 1-space bounded cube packing
}

\author{
Łukasz Zielonka \\ Institute of Mathematics and Physics \\ UTP University of Science and Technology \\ Al. Prof. S. Kaliskiego 7, 85-789 Bydgoszcz, Poland \\ Email: Lukasz.Zielonka@utp.edu.pl
}

\begin{abstract}
In this paper, we present a 1-space bounded cube packing algorithm with asymptotic competitive ratio 10.872 .

Index Terms-Online algorithms, bin packing, cube, one-space bounded
\end{abstract}

\section{INTRODUCTION}

$\mathbf{I}$ $\mathrm{N}$ THE bin packing problem, we receive a sequence of items of different sizes that must be packed into a finite number of bins in a way that minimizes the number of bins used. When all the items are accessible, the packing method is called offline. The packing method is called online, when items arrive one by one and each item has to be packed irrevocably into a bin before the next item is presented.

In the online version of packing a crucial parameter is the number of bins available for packing, i.e., active bins. Each incoming item is packed into one of the active bins; the remaining bins are not available at this moment. If we close one of the current active bins, we open a new active bin. Once an active bin has been closed, it can never become active again. When the method allows at most $t$ active bins at the same time, it is called $t$-space bounded. Unbounded space model does not impose any limits on the number of active bins. It is natural to expect a packing method to be less efficient with fewer number of active bins. In this paper, we study 1-space bounded 3-dimensional cube packing.

Let $S$ be a sequence of cubes. Denote by $A(S)$ the number of bins used by the algorithm $A$ to pack items from $S$. Furthermore, denote by $O P T(S)$ the minimum possible number of bins used to pack items from $S$ by the optimal offline algorithm. By the asymptotic competitive ratio for the algorithm $A$ we mean:

$$
R_{A}^{\infty}=\limsup _{n \rightarrow \infty} \sup _{S}\left\{\frac{A(S)}{O P T(S)} \mid \operatorname{OPT}(S)=n\right\} .
$$

\section{A. Related work}

The one-dimensional case of the space bounded bin packing problem has been extensively studied and the best possible algorithms are known: the Next-Fit algorithm [5] for the onespace bounded model and the Harmonic algorithm [6] when the number of active bins goes to infinity. The questions concerning $t$-space bounded $d$-dimensional packing $(d \geq 2)$ have been studied in a number of papers. For large number of active bins, Epstein and van Stee [1] presented a $\left(\Pi_{\infty}\right)^{d}$. competitive space bounded algorithm, where $\Pi_{\infty} \approx 1.69103$ is the competitive ratio of the one-dimensional algorithm
Harmonic. Algorithms for 2-dimensional bin packing with only one active bin were explored for the first time in [8], where the authors give 8.84-competitive algorithm for 2dimensional bin packing. An improved result of that case can be found in the paper [7], where a 5.155-competitive method is presented. The last article also contains an algorithm for packing squares with competitive ratio at most 4.5. In [4], a 4.84-competitive 1-space bounded 2-dimensional bin packing algorithm was presented. Grzegorek and Januszewski [3] presented a $3.5^{d}$-competitive as well as a $12 \cdot 3^{d}$-competitive online $d$-dimensional hyperbox packing algorithm with one active bin. The $d$-dimensional case of 1-space bounded hypercube packing was discussed in [9], where a $2^{d+1}$-competitive algorithm was described. The aim of this paper is to improve the upper bound $\left(2^{3+1}\right)$ in the 3 -dimensional case. We present 10.872-competitive 1-space bounded cube packing algorithm.

\section{B. Our results}

The algorithm presented in this article considers packing items (cubes of edges not greater than 1) into one active cube of edge 1 . The main packing method is a bit like the classic computer game Tetris. The packing method which we describe is similar to the method presented by Grzegorek and Januszewski in [2]. The algorithm distinguishes types of items what determines a method for packing a specific item in a bin. Items that are considered big enough are packed from top to bottom. Different types of small items are packed from bottom upwards. The algorithm handles small items in a Tetris manner: to determine a place to pack an item a part of a bin is temporarily divided into congruent cuboids of appropriate size. Then an item is packed as low as possible inside a carefully chosen cuboid.

In Section II we give a 1-space bounded cube packing algorithm with the ratio 10.872 .

\section{THE one-space-ALGORITHM}

Let $S$ be a sequence of cubes $Q_{1}, Q_{2}, \ldots$ Denote by $a_{i}$ the edge length of $Q_{i}$.

- an item $Q_{i}$ is huge, provided $a_{i}>1 / 2$;

- an item $Q_{i}$ is big, provided $1 / 4<a_{i} \leq 1 / 2$;

- an item $Q_{i}$ is small, provided $a_{i} \leq 1 / 4$; a small item $Q_{i}$ is of type $k$ provided $2^{-k-1}<a_{i} \leq 2^{-k}$.

Let $\mathcal{B}$ be the active bin. To shorten the notation, a cuboid whose edges have lengths $a \times a \times b$ will be called an $(a, b)$ cuboid. 


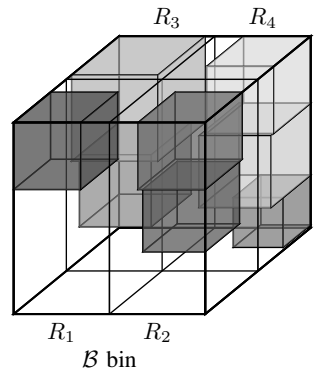

Fig. 1. Big items - the darker an item's colour, the later it arrived

\section{A. Description of the one-space-algorithm}

(a) In packing items we distinguish coloured and white (not coloured) space. Items are placed only in the white space. Each newly opened bin is white.

(b) We divide each freshly opened bin into $(1 / 2,1)$-cuboids. These cuboids are named $R_{1}, R_{2}, R_{3}, R_{4}$ in an arbitrary order.

(c) Huge items (edge $>1 / 2$ ) are packed alone into a bin, i.e., if $Q_{i}$ is huge, then we close the active bin and open a new bin to pack this item. After packing $Q_{i}$ we close the bin and open a new active bin.

(d) If $Q_{i}$ is big $(1 / 4<$ edge $\leq 1 / 2)$ we find the highest indexed $R_{j}$ such that $Q_{i}$ can be packed into it. We pack $Q_{i}$ into $R_{j}$ along the edge of $\mathcal{B}$ as high as it is possible (see Figs. 1 and 3 ). If such a packing is not possible, we close the active bin, open a new active bin and pack $Q_{i}$ into it.

When a big item is packed, it colours the space covered by itself.

(e) If $Q_{i}$ is a small item of type $k\left(2^{-k-1}<\right.$ edge $\left.\leq 2^{-k}\right)$ (see Figs. 2 and 3 ) we find the lowest indexed $R_{j}$ such that $Q_{i}$ can be packed into it. Since $j$ is fixed now, we will write $R$ instead of $R_{j}$.

We temporarily divide $R$ into $\left(2^{-k}, 1\right)$-cuboids called $R(1), \ldots, R\left(4^{k-1}\right)$. Denote by $t(n)$ the distance between the top of $R(n)$ and the top of the topmost item packed in $R(n)$ for $n=1, \ldots, 4^{k-1}$ (see Fig. 5, right) and let $\eta$ be an integer such that $t(\eta)=\max \left\{t(1), \ldots, t\left(4^{k-1}\right)\right\}$. We pack $Q_{i}$ into $R(\eta)$ as low as possible. The result of packing $Q_{i}$ is the colouring of the $\left(2^{-k}, 1-t(\eta)+a_{i}\right)$ cuboid contained in the bottom of $R(\eta)$ (see Fig. 5, right, where $\eta=2$ before $Q_{14}$ was packing).

If such a packing is not possible, then we close the active bin and open a new active bin to pack $Q_{i}$.

\section{B. Competitive ratio}

Let $P_{j}$ for $j=1, \ldots, 16$ be $(1 / 4,1)$-cuboids with pairwise disjoint interiors. Each cuboid $R_{i}$ for $i \in\{1,2,3,4\}$ is divided into four cuboids $P_{4 i-3}, \ldots, P_{4 i}$ (see Fig. 4).

Lemma 1. Assume that only small items were packed into $\mathcal{B}$. Assume that $j \in\{1,2, \ldots, 16\}$. Denote by $n$ the number of items packed into $P_{j}$ and by $t_{n}$ the distance between the bottom of $\mathcal{B}$ and the top of the topmost item packed into $P_{j}$. The total volume $v_{n}$ of small items packed into $P_{j}$ is greater than

$$
f\left(t_{n}\right)=\frac{19}{2048} \cdot t_{n}-\frac{13}{16384} .
$$

Moreover, if the topmost packed item is of type 2, then

$$
v_{n}>f_{+}\left(t_{n}\right)=\frac{19}{2048} \cdot t_{n}
$$

Proof. Without loss of generality we can assume that $P_{j}=$ $[0,1 / 4] \times[0,1 / 4] \times[0,1]$. We will prove the result using induction over the number $n$ of packed items.

First assume that only one item $Q_{b}$ was packed into $P_{j}$. Obviously, $t_{1}=a_{b}$. Let

$$
\varphi(a)=a^{3}-\frac{19}{2048} a .
$$

The function $\varphi(a)$ for $a>0$ has a minimum at

$$
a_{0}=\sqrt{\frac{19}{6144}} \text {. }
$$

A computation shows that

$$
\varphi\left(a_{0}\right)>-\frac{1}{2} \cdot \frac{13}{16384}
$$

(this lower bound will be useful in the last part of the proof). We get

$$
v_{1}=a_{b}^{3}>\frac{19}{2048} \cdot t_{1}-\frac{1}{2} \cdot \frac{13}{8192}=f\left(t_{1}\right) .
$$

Moreover, if $1 / 8<a_{b} \leq 1 / 4$, then $v_{1}=a_{b}^{3}>\frac{19}{2048} a_{b}=$ $f_{+}\left(t_{1}\right)$.

Now assume that the statement holds for at most $n$ items packed into $P_{j}$ (this is our inductive assumption). Let $Q_{u}$ be the $(n+1)$ st item packed into $P_{j}$ and let $t_{n+1}$ be the distance between the bottom of $P_{j}$ and the top of the topmost item (from among $n+1$ items $Q_{b}, \ldots, Q_{u}$ ) packed into $P_{j}$.

If $a_{u}>1 / 8$, then $t_{n+1}=t_{n}+a_{u}$. Using the inductive assumption,

$$
v_{n+1}=v_{n}+a_{u}^{3}>f\left(t_{n}\right)+a_{u}^{3}=\frac{19}{2048} \cdot t_{n}-\frac{13}{16384}+a_{u}^{3} .
$$

Since

$$
\varphi^{\prime}(a)=3 a^{2}-\frac{19}{2048}>3 \cdot \frac{1}{64}-\frac{19}{2048}>0
$$

for $a>1 / 8$, we get

$$
\varphi(a)>\varphi\left(\frac{1}{8}\right)=\frac{13}{16384}
$$

for $a>1 / 8$. Consequently,

$$
\begin{aligned}
v_{n+1} & >f\left(t_{n}\right)+a_{u}^{3}=\frac{19}{2048}\left(t_{n}+a_{u}\right)+\varphi\left(a_{u}\right)-\frac{13}{16384} \\
& \geq \frac{19}{2048}\left(t_{n}+a_{u}\right)=f_{+}\left(t_{n}+a_{u}\right)=f_{+}\left(t_{n+1}\right) .
\end{aligned}
$$

Finally, consider the case when $a_{u} \leq 1 / 8$. First, we choose the topmost packed item $Q^{1}$ with edge greater than $1 / 8$ and denote by $\tau$ the distance between the bottom of $P_{j}$ and the top of $Q^{1}$ (see Fig. 6, left). If there is no such item, then we 


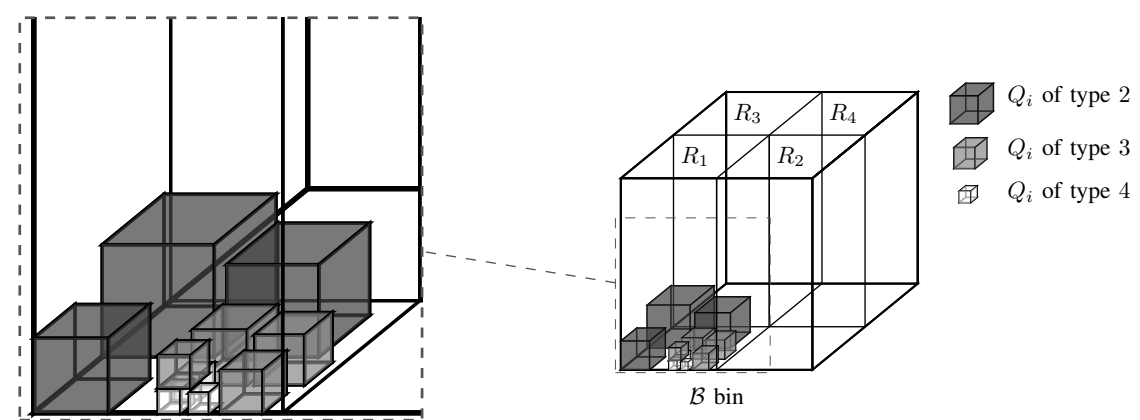

Fig. 2. Small items

take $\tau=0$. The total volume of items packed up to $\tau$, by the inductive assumption, is not smaller than $f_{+}(\tau)$. Above $Q^{1}$ we divide $P_{j}$ into four $(1 / 8,1-\tau)$-cuboids $P_{j}^{1}, P_{j}^{2}, P_{j}^{3}, P_{j}^{4}$. Denote by $Q_{1}^{l}, \ldots, Q_{v_{l}}^{l}$ the items from among $Q_{b}, \ldots, Q_{u-1}$ packed into $P_{j}^{l}$ above $Q^{1}$ (if any) for each $l=1,2,3,4$. Moreover, denote by $t_{n}^{l}$ the distance between the bottom of $P_{j}$ and the top of the topmost item from among $Q_{b}, \ldots, Q_{u-1}$ packed into $P_{j}^{l}$ and let $t_{n}^{*}=\min \left(t_{n}^{1}, t_{n}^{2}, t_{n}^{3}, t_{n}^{4}\right)$ (see Fig. 6, right). Clearly, $t_{n}^{*} \geq \tau$ and $t_{n}^{*} \leq t_{n}$.

If $t_{n}^{*}+a_{u} \leq t_{n}$, then $t_{n+1}=t_{n}$. Consequently,

$v_{n+1} \geq v_{n}+a_{u}^{3}=f\left(t_{n}\right)+a_{u}^{3}=f\left(t_{n+1}\right)+a_{u}^{3}>f\left(t_{n+1}\right)$.

If $t_{n}^{*}+a_{u}>t_{n}$, then $t_{n+1}=t_{n}^{*}+a_{u}$. Items $Q_{1}^{l}, \ldots, Q_{v}^{l}$ were packed into $\left(1 / 8, t_{n}^{l}-\tau\right)$-cuboid $P_{j}^{l}$. Let $h\left(P_{j}^{l}\right)=$ $[0,1 / 4] \times[0,1 / 4] \times\left[0,2 t_{n}^{l}-2 \tau\right]$ be the image of $P_{j}^{l}$ in a homothety $h$ of ratio 2 . By the inductive assumption, the total volume of cubes $h\left(Q_{1}^{l}\right), \ldots, h\left(Q_{v}^{l}\right)$ is not smaller than $\frac{19}{2048}\left(2 t_{n}^{l}-2 \tau\right)-\frac{13}{16384}=f\left(2 t_{n}^{l}-2 \tau\right)$. Since the volume of each $h\left(Q_{i}^{l}\right)$ is 8 times greater than the volume of $Q_{i}^{l}$, it follows that the total volume of cubes $Q_{1}^{l}, \ldots, Q_{v}^{l}$ is not smaller than $\frac{1}{8} f\left(2 t_{n}^{l}-2 \tau\right)$.

Consequently,

$$
\begin{aligned}
v_{n+1} & \geq f_{+}(\tau)+4 \cdot \frac{1}{8} f\left(2 t_{n}^{*}-2 \tau\right)+a_{u}^{3} \\
& =a_{u}^{3}+\frac{19}{2048} t_{n}^{*}-\frac{1}{2} \cdot \frac{13}{16384} .
\end{aligned}
$$

By (1) we know that

$$
\varphi\left(a_{0}\right)>-\frac{1}{2} \cdot \frac{13}{16384} .
$$

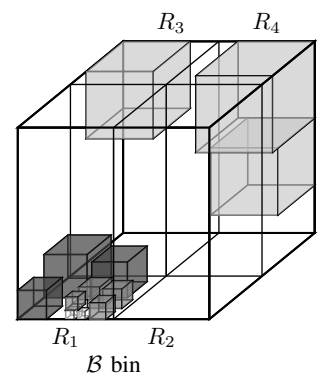

Fig. 3. one-space-algorithm
Consequently,

$$
\begin{aligned}
v_{n+1} & \geq \varphi\left(a_{u}\right)+\frac{19}{2048}\left(t_{n}^{*}+a_{u}\right)-\frac{1}{2} \cdot \frac{13}{16384} \\
& >\frac{19}{2048}\left(t_{n}^{*}+a_{u}\right)-\frac{13}{16384}=f\left(t_{n+1}\right) .
\end{aligned}
$$

Lemma 2. Define $V_{3}=101 / 1024$. Let $S$ be a finite sequence of cubes and let $\nu$ be the number of bins used to pack items from $S$ by the one-space-algorithm. Moreover, let $m$ be the number of huge items in $S$. The total volume of items in $S$ is greater than $2^{-3} \cdot m+V_{3}(\nu-2 m-1)$.

Proof. Among $\nu$ bins used to pack items from $S$ by the one-space-algorithm the first $\nu-1$ bins will be called full. Let $Q_{z}$ be the first item from $S$ which cannot be packed into a full bin $\mathcal{B}$ by the one-space-algorithm. Clearly, $Q_{z}$ is the first item packed into the next bin.

Denote by $v_{\mathcal{B}}$ the sum of volumes of items packed into $\mathcal{B}$.

If the incoming item $Q_{z}$ is huge, then the average occupation ratio in both bins $\mathcal{B}_{j}$ and the next bin $\mathcal{B}_{j+1}$ into which $Q_{z}$ was packed is greater than $1 / 2^{4}$. Obviously, there are $2 m$ such bins.

It is possible that the last bin is almost empty.

To prove Lemma 2 it suffices to show that if $Q_{z}$ is not huge and if no huge item was packed into $\mathcal{B}$, then $v_{\mathcal{B}}>V_{3}$ (the number of such bins equals $\nu-2 m-1$ ).

Case 1: $Q_{z}$ is small and all items packed into $\mathcal{B}$ are small.

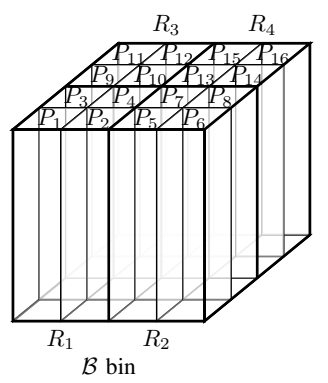

Fig. 4. (1/4,1)-cuboids $P_{j}$ 

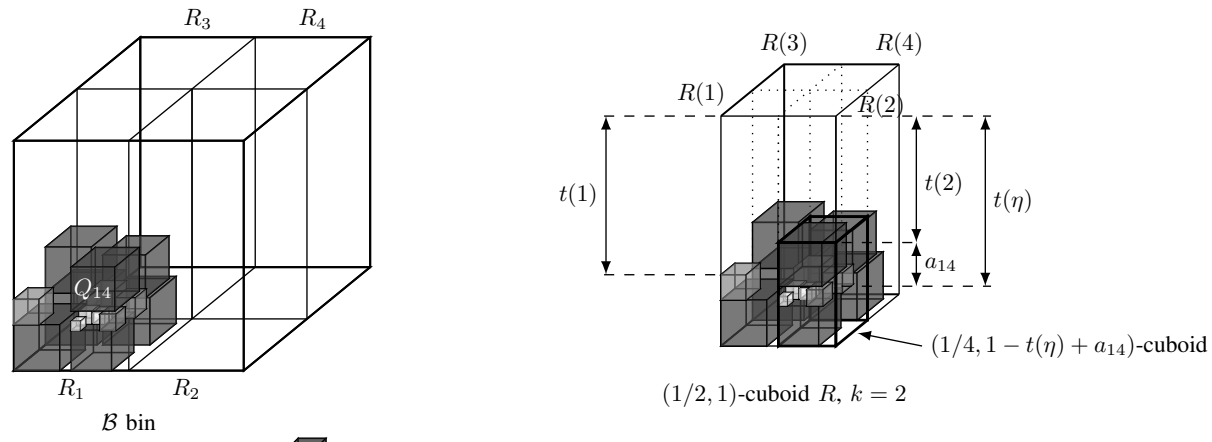

$(1 / 2,1)$-cuboid $R, k=2$

$Q_{i}$ of type 2

$Q_{i}$ of type $3 \quad$ Q $Q_{i}$ of type 4

Fig. 5. Packing small items into $\left(2^{-k}, 1\right)$-cuboids

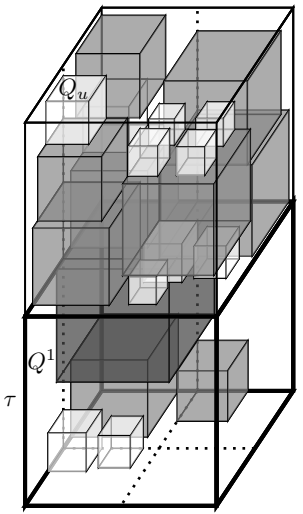

$Q_{i}$ of type 2

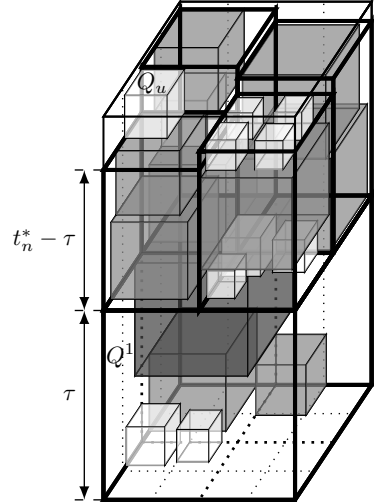

$Q_{i}$ of type $3 \quad Q_{i}$ of type 4
Fig. 6. The division

Since $a_{z} \leq 1 / 4$, it follows that each $P_{i}$ is packed up to height at least $3 / 4$. By Lemma 1 we deduce that

$$
v_{\mathcal{B}}>4^{2} f\left(\frac{3}{4}\right)=16 \cdot\left(\frac{19}{2048} \cdot \frac{3}{4}-\frac{13}{4 \cdot 16384}\right)=V_{3} .
$$

Case 2: $Q_{z}$ is small and a big item was packed into $\mathcal{B}$.

The volume of a big item $Q_{b}$ with edge $t$ is equal to $t^{3}>$ $t \cdot\left(\frac{1}{4}\right)^{2}$. In considerations presented in Case 1 we accept that the total volume of small items packed into $R_{j}$ up to height $t$ equals $4 f(t)$. It is easy to see that

$$
4 f(t)<\frac{1}{16} \cdot t
$$

As a consequence, $v_{\mathcal{B}}>V_{3}$.

Case 3: $Q_{z}$ is a big item and all items packed into $\mathcal{B}$ are small

Assume that there is $\left(2^{-2}, 1\right)$-cuboid $R_{j}(n) \quad(j \in$ $\{1,2,3\}, n \in\{1,2,3,4\})$ such that the distance between its top and the top of the topmost item packed into it is greater than $1 / 8$ and denote by $R_{+}$first such cuboid. The total volume of items packed into $R_{+}$is greater than $f(3 / 4)$. The total volume of items packed into each cuboid preceding $R_{+}$is greater than $f(7 / 8)$. The total volume of items packed into

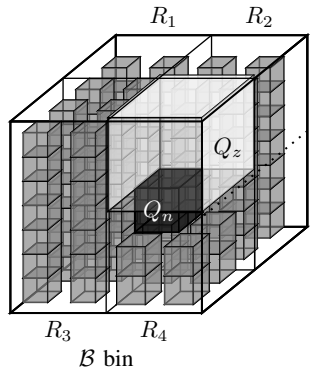

Fig. 7. Case 3

each of remaining cuboids is greater than $\frac{3}{4} \cdot \frac{1}{8^{2}}>f(7 / 8)$ (in such a cuboid only items greater than $1 / 8$ were packed).

Denote by $Q_{n}$ the topmost small item packed in $R_{4}$ (as in Fig. 7). Since $a_{z} \leq 1 / 2$ and $Q_{z}$ cannot be packed in $R_{4}$, it follows that

$$
v_{\mathcal{B}}>(16-5) f\left(\frac{7}{8}\right)+f\left(\frac{3}{4}\right)+4 f\left(\frac{1}{2}-a_{n}\right)+a_{n}^{3} .
$$

Denote by $\gamma\left(a_{n}\right)$ the function on the right-hand side of this formula. This function for positive $a$ has a minimum at $a_{0}=$ $\sqrt{\frac{19}{1536}}$.

A computation shows that $\gamma\left(a_{0}\right)>V_{3}$. Consequently, $v_{\mathcal{B}}>V_{3}$.

If there is no $\left(2^{-2}, 1\right)$-cuboid $R_{j}(n)(j \in\{1,2,3\}, n \in$ $\{1,2,3,4\})$ such that the distance between its top and the top of the topmost item packed into it is greater than $1 / 8$, then

$$
v_{\mathcal{B}}>(16-4) f\left(\frac{7}{8}\right)+4 f\left(\frac{1}{2}-a_{n}\right)+a_{n}^{3} .
$$

Since $f(7 / 8)>f(3 / 4)$, we get $v_{\mathcal{B}}>\gamma\left(a_{0}\right)>V_{3}$.

Case 4: $Q_{z}$ is big and a big item was packed into $\mathcal{B}$

Similarly as in Case 2 we get

$$
4 f(t)<t^{3} .
$$

We deduce by Case 3 that $v_{\mathcal{B}}>V_{3}$. 
Theorem 1. The asymptotic competitive ratio for the one-space-algorithm is not greater than 1098/101 $\approx$ 10.8713 .

Proof. Let $S$ be a sequence of items of total volume $v$, let $m$ denote the number of huge items in $S$ and let $\mu$ be the number of bins used to pack items from $S$ using the one-space-algorithm. Obviously, $\operatorname{OPT}(S) \geq v$ as well as $O P T(S) \geq m$.

By Lemma 2 we get $v>\frac{1}{2^{3}} \cdot m+V_{3} \cdot(\mu-2 m-1)$, i.e.,

$$
\mu<\frac{v}{V_{3}}+m\left(2-\frac{1}{2^{3} V_{3}}\right)+1
$$

It is easy to check that $2-\frac{1}{8 V_{3}}>0$.

If $m<v$, then

$\frac{\mu}{O P T(S)} \leq \frac{\mu}{v}<\frac{\frac{v}{V_{3}}+v\left(2-\frac{1}{2^{3} V_{3}}\right)+1}{v}=\frac{2^{3}-1}{2^{3} V_{3}}+2+\frac{1}{v}$.

If $v \leq m$, then

$\frac{\mu}{O P T(S)} \leq \frac{\mu}{m} \leq \frac{\frac{m}{V_{3}}+m\left(2-\frac{1}{2^{3} V_{3}}\right)+1}{m}=\frac{2^{3}-1}{2^{3} V_{3}}+2+\frac{1}{m}$.

Consequently, the asymptotic competitive ratio for the one-space-algorithm is not greater than

$$
\frac{7}{8} \cdot \frac{1024}{101}+2=\frac{1098}{101}<10.872 .
$$

\section{REFERENCES}

[1] L. Epstein and R. van Stee. Optimal online algorithms for multidimensional packing problems. SIAM Journal on Computing, 35(2):431-448, 2005.

[2] P. Grzegorek and J. Januszewski. A note on one-space bounded square packing. Information Processing Letters, 115(11):872-876, 2015.

[3] P. Grzegorek, J. Januszewski. Drawer algorithms for 1-space bounded multidimensional hyperbox packing. Journal of Combinatorial Optimization, 37(3): 1011-1044, 2019.

[4] J. Januszewski and $€$. Zielonka. Online packing of rectangular items into square bins. In R. Solis-Oba and R. Fleischer, editors, Approximation and Online Algorithms. WAOA 2017, volume 10787 of Lecture Notes in Computer Science, pages 147-163, Cham, 2018. Springer.

[5] D. S. Johnson. Fast algorithms for bin packing. Journal of Computer and System Sciences, 8(3):272-314, 1974.

[6] C.-C. Lee and D.-T. Lee. A simple on-line bin-packing algorithm. J. ACM, 32(3):562-572, July 1985.

[7] Y. Zhang, J. Chen, F. Y. L. Chin, X. Han, H.-F. Ting, and Y. H. Tsin. Improved online algorithms for 1-space bounded 2-dimensional bin packing. In O. Cheong, K.-Y. Chwa, and K. Park, editors, Algorithms and Computation, pages 242-253, Berlin, Heidelberg, 2010. Springer.

[8] Y. Zhang, F. Y. L. Chin, and H.-F. Ting. One-space bounded algorithms for two-dimensional bin packing. International Journal of Foundations of Computer Science, 21(06):875-891, 2010.

[9] Y. Zhang, F. Y. L. Chin, H.-F. Ting, and X. Han. Online algorithms for 1 -space bounded multi dimensional bin packing and hypercube packing. Journal of Combinatorial Optimization, 26(2):223-236, 2013. 